Progress in Propulsion Physics 4 (2013) 33-58

DOI: $10.1051 /$ eucass/201304033

(C) Owned by the authors, published by EDP Sciences, 2013

\title{
ANALYSIS AND SYNTHESIS OF SOLUTIONS FOR THE AGGLOMERATION PROCESS MODELING
}

\author{
V. A. Babuk, I. N. Dolotkazin, and A. A. Nizyaev \\ Department of Space Vehicles and Rocket Motors \\ Baltic State Technical University \\ 1 First Krasnoarmeyskaya Str., St.-Petersburg 190005, Russia
}

The present work is devoted development of model of agglomerating process for propellants based on ammonium perchlorate (AP), ammonium dinitramide (ADN), HMX, inactive binder, and nanoaluminum. Generalization of experimental data, development of physical picture of agglomeration for listed propellants, development and analysis of mathematical models are carried out. Synthesis of models of various phenomena taking place at agglomeration implementation allows predicting of size and quantity, chemical composition, structure of forming agglomerates and its fraction in set of condensed combustion products. It became possible in many respects due to development of new model of agglomerating particle evolution on the surface of burning propellant. Obtained results correspond to available experimental data. It is supposed that analogical method based on analysis of mathematical models of particular phenomena and their synthesis will allow implementing of the agglomerating process modeling for other types of metalized solid propellants.

\section{INTRODUCTION}

Usage of metal fuel (aluminum) in propellant composition has a consequence formation of condensed combustion products. As a rule, the set of condensed products includes two fractions. The first one consist of smoke oxide particles (SOP) having size typically not more than $1 \mu \mathrm{m}$. The second one includes set of agglomerates formed due to condensed products enlargement processes in surface layer of burning propellant. Agglomerates could exceed initial metal particles sizes in 10-1000 times (agglomerates more than $1 \mathrm{~mm}$ in diameter are experimentally obtained [1]). 
The presence of agglomerates in set of combustion products results in appearance of problems associated with efficiency of rocket motor working. These include slag accumulation, two-phase and chemical losses of specific impulse, erosion of thermal protection, and increased heat stress on the elements of rocket motor. In should be noted that there are experimental data of positive effect of agglomerating process on the propellant burning law [2]. This effect consists in reduction of dependence of burning rate from pressure.

Commonly, the data of size of condensed particles are used only at calculations of two-phase flows and this influence on work of rocket motor. However, in several papers (see, for example, [3]), it is concluded that information about the chemical composition and structure of condensed particles is necessary. The value of unoxidized aluminum in the composition of depositing agglomerates is important information for description of interaction between two-phase flows and ablative materials [4]. Structure of agglomerates and especially presence of gaseous cavities has significant influence on particles behavior in the gaseous flows [5]. Thus, knowledge of amount of agglomerates in the set of combustion products, chemical composition, and structure of agglomerates is necessary besides of sizes of agglomerates for the description of agglomeration process and its influence on quality of the system "propellant-rocket motor."

Let consider decisions that have been used for modeling of agglomeration process in earlier published papers. The extensive review of these works has been carried out by Beackstead [6]. Apparently, only the information on the works carried out last years in SPLab Politecnico di Milano [7, 8] is absent in this paper.

Used decisions provide the account of influence on agglomeration of propellant structure and pressure in the chamber (burning rate of propellant).

These decisions can be divided conditionally into three groups:

(1) decisions that are based on application of "pocket" model [9]. They are used in [10-12]. Gallier named "pocket" a congestion of initial particles of metal in propellant [13]. The similar approach is also applied in a number of other papers $\left[8,14^{*}\right]$. At use of "pocket" model, the size of agglomerates is defined only by propellant structure;

(2) decisions that are based on consideration of conditions of a separation of agglomerating particles from a burning surface, which are connected with the burning process [15-20]; and

(3) decisions that are a combination of decisions of the first and second groups, i. e., influence on agglomeration process as propellant structure, and burning process are considered $\left[14^{\dagger}, 21-28\right]$.

\footnotetext{
${ }^{*}$ Model A.

${ }^{\dagger}$ Models B1 and B2.
} 
The developed models provide determination of the characteristic sizes of the agglomerates. Some models allow to determine additionally the function of size of agglomerates distribution. Besides, some models give the chance to find such important characteristic of process of agglomeration as a fraction of agglomerating metal $[6,13,14]$. It is necessary to mention the increased attention during last years to numerical modeling of distribution of a disperse phase in propellants $[7,29-31]$. Thus, as a rule, jammed packs are considered. The results of such modeling are used by working out of some agglomeration models.

Generally, the question on adequacy of modeling remains open. Comparison of calculated and experimental data either was not carried out, or carried out for specific propellants in some conditions using the coefficients matching. Besides, a number of positions of models contradict experimental data. In particular, "pocket" mechanism of agglomeration is realized only in come conditions, ignition of agglomerating particles does not always define conditions of a breaking away of particles from a surface of burning propellant. As a whole, it is possible to say that available models describe the general tendencies of influence of various factors for the size of agglomerates for specific propellants.

The authors of the present paper at the agglomeration description use the concept of a skeleton layer (SL). The SL is a gas-permeable three-dimensional structure consisting mainly of the metal and its oxide, as well as thermostable carbonic elements, and comprising the top portion of surface layer. The results of numerous experimental researches [1,2,32-37] give the grounds to assert that properties of this layer define all characteristics of agglomerates (fraction in combustion products, size, chemical composition, and structure). Presence of the SL ensures long-time residence, movement, merging, and enlargement of hightemperature agglomerating particle in the surface layer of burning propellant.

Change of parameters of propellant composition (a kind, content and size of components) has as a consequence change of properties of SL, and, respectively, of agglomerates. These changes can have rather significant (dramatical) character. The size of agglomerates can change from 10 to $1000 \mu \mathrm{m}$, a mass fraction in condensed combustion products - from several percent to $80 \%-95 \%$, content of oxide in agglomerates - from several to $85 \%$, the structure of agglomerates can be various: a metal particle with "cap" oxide, "matrix" agglomerates, "hollow" agglomerates, a particle of metal with an oxide layer $[1,2,32-37]$. Thus, it is possible to speak about variety of displays of agglomeration process. This circumstance gives illusory possibility of working out of the universal description considering all features of agglomeration process at burning of those or other types of propellants in various conditions. As considered above, there exists rather expedient separation of classes and types of propellants, at which burning close laws are realized.

Depending on the SL properties, the propellants were divided into two classes - A and B [34]. For burning propellants of class A, the metal ignition temperature is less than the decomposition temperature of carbonaceous 
elements. The SL of these propellants consists of a carbon skeleton with pores filled by liquid metal and oxide. On the contrary, for burning propellants of class $\mathbf{B}$, the metal ignition temperature is higher than the decomposition temperature of carbonaceous elements. The SL of these propellants consists of the initial metal particles fastened among themselves. The results of paper [38] allow to define how various decisions on propellant composition (active-inactive binder, aluminum-nanoaluminum, oxidizers: AP, ADN, ammonium nitrate (AN), HMX) influence on the accessory of propellants to one or another class and the law of their burning.

The purpose of the present paper was working out of the mathematical model providing definition of a complex of characteristics of agglomerates:

- function of size agglomerates distribution;

- fraction of agglomerates in combustion products; and

- parameters of a chemical composition and structure of agglomerates.

As to the present authors' knowledge, the similar problem was not solved earlier.

So, one of types of propellants for the decision of a problem of modeling has been chosen. This type includes propellants on the base of AP, ADN, HMX, inactive binder, and nanoaluminum. It is supposed that metal particles in fuel keep the individuality. These propellants belong to the propellants of class A and have similar regularities of agglomeration process [34]. It is necessary to note that these propellants can have rather important positive property - weak dependence of burning rate on pressure [2].

\section{PHYSICAL PICTURE OF THE AGGLOMERATION PROCESS}

Physical picture of the agglomeration process at burning of considered propellants is formulated on the basis of available by this time experimental data analysis. The experiments were performed with use of the following techniques [34, 37]:

- quench-collection of condensed combustion products at combustion of propellant samples and subsequent chemical and structural analysis at a variation of parameters of propellant composition, pressure, value, and direction of accelerative forces;

- investigations of combustion residues or quenching products; and

- visualization of the surface of burning propellant. 
The first method allows obtaining quantitative data of size, chemical composition and structure of agglomerates. Most important of them are:

$f_{m}(D)$ - mass function of size distribution density of agglomerates;

$Z_{m}$ - fraction of unburned metal in agglomerates relative to initial metal in propellant;

$Z_{m}^{\text {ox }}$ - fraction of initial metal in propellant used to form oxide in agglomerates;

$Z_{m}^{a}$ - fraction of initial metal in propellant used to form agglomerates;

$\eta$ - mass fraction of oxide in agglomerate; and

$D_{43}$ — mass-medium diameter of agglomerates, $\mu \mathrm{m}$.

Both other methods provide obtaining qualitative information describing agglomerating particles behavior on the surface of burning propellant and in the near-surface zone of gas phase.

Analysis of experimental data allowed to indicate following stages of agglomeration process.

\subsection{Formation of the Skeleton Layer}

As it was specified earlier, forming of the SL is the necessary condition for the agglomeration process realization. First of all, SL provides coherence of initial particles of metal and products of their transformation that is an important condition of agglomeration.

Occurrence SL at propellant burning is possible only at formation of a carbon skeleton [36, 38]. Consequently, the possibility of the carbon

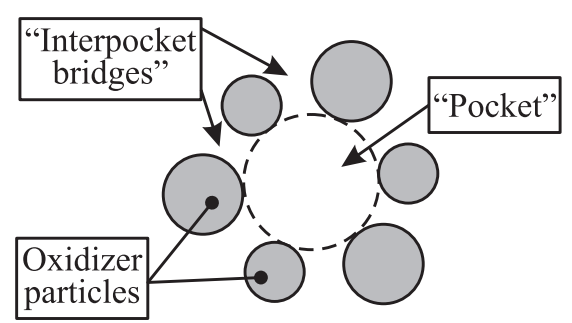

Figure 1 The local area of propellant containing a "pocket" skeleton formation and SL is connected with conditions of the binder decomposition — first of all, with oxidizing components concentration. Formation of the SL for considered propellants occurs only within the "pockets" (Fig. 1). Within other structural formations "interpocket bridges" which are characterized by increased oxidizing components concentration - formation of the SL does not occur. It should be noted that the usage of active binders leads to increase of oxidizing components as well as within the "pockets" which, in turn, leads to suppression of the agglomeration process [36]. 
Thus, formation of the SL for the considered propellants is connected with presence of the "pockets." Therefore, for such propellants, there is the dependence between fraction of initial metal in propellant used to form agglomerates (parameter $Z_{m}^{a}$ ) and propellant structure.

\subsection{Ignition and Burning of Metal}

The combustion of considered propellants corresponds to regularities of the class A propellants burning [34], thus the temperature of metal ignition does not exceed the decomposition temperature of thermostable carbonic elements of the SL. Gas permeability of the SL structure ensures the access for oxidizing components inside the SL. The presence of solid oxide film does not preclude ignition, since oxidizing gases are able to penetrate through the oxide film cracks. Rather low value of ignition temperature is defined by use of nanoaluminum.

After ignition of metal, oxide is melting relatively quickly. This event ensures the possibility of spreading of liquid metal and oxide inside the SL. It is considered that existence of chemical interaction between these substances and carbon ensures the liquid metal and oxide wetting of carbonic elements. Therefore, these liquid substances fill the SL pores.

After ignition, the metal is burning in the heterogeneous mode, and it is accompanied by accumulation of liquid oxide. Such burning mode continues until the temperature of the metal particles reaches the value required for the heterogeneous mode to transfer to the gas-phase mode. At the same time, accumulation of liquid oxide is terminated, and product of combustion of metal in this mode is the SOP carried away by the gas flow [39].

\subsection{Formation of Individual Agglomerating Particles}

During combustion of the "pocket," liquid metal and oxide are moving up to the surface of SL and forming the particle staying at the I equilibrium state (Fig. 2). At this state, breaking away of the particle is not possible due to high level of adhesion between particles substance and elements of the SL [24]. As the "pocket" burns out, the volume of the drop on the SL surface increases.

The picture changes in the case of macroinhomogeneity appearing, which is the oxidizer particle. In that case, the nonequilibrium state appears, and the particle is transferring to the new equilibrium state - the II equilibrium state [24]. At this state, breaking away of the particle becomes possible. Transferring of the particles state is accompanied with the change of metal burning mode - from the heterogeneous to the gas phase. Connection of the agglomerating particle with the SL elements due to capillary forces ensures residence of the particle in this state for some time. The value of this time is significantly less than the time 


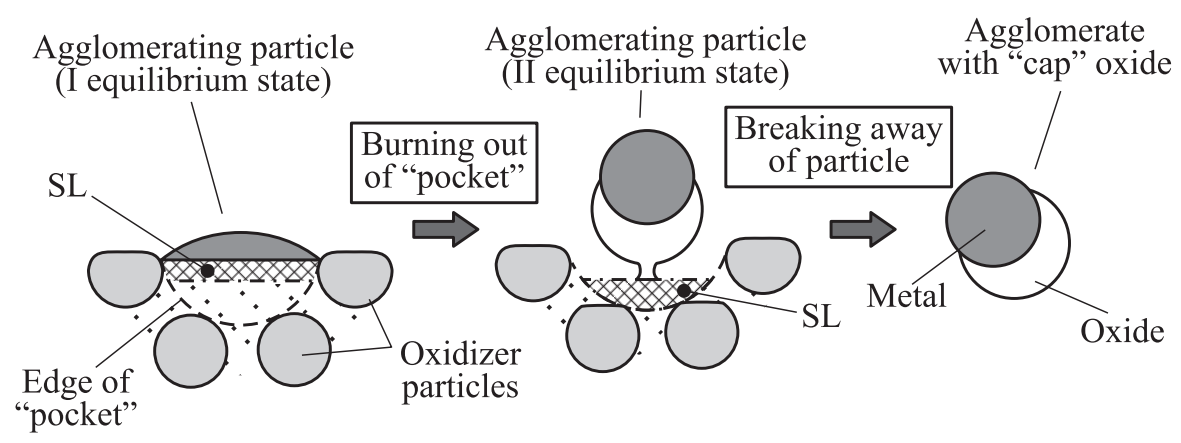

Figure 2 Scheme of the "pocket" agglomeration mechanism

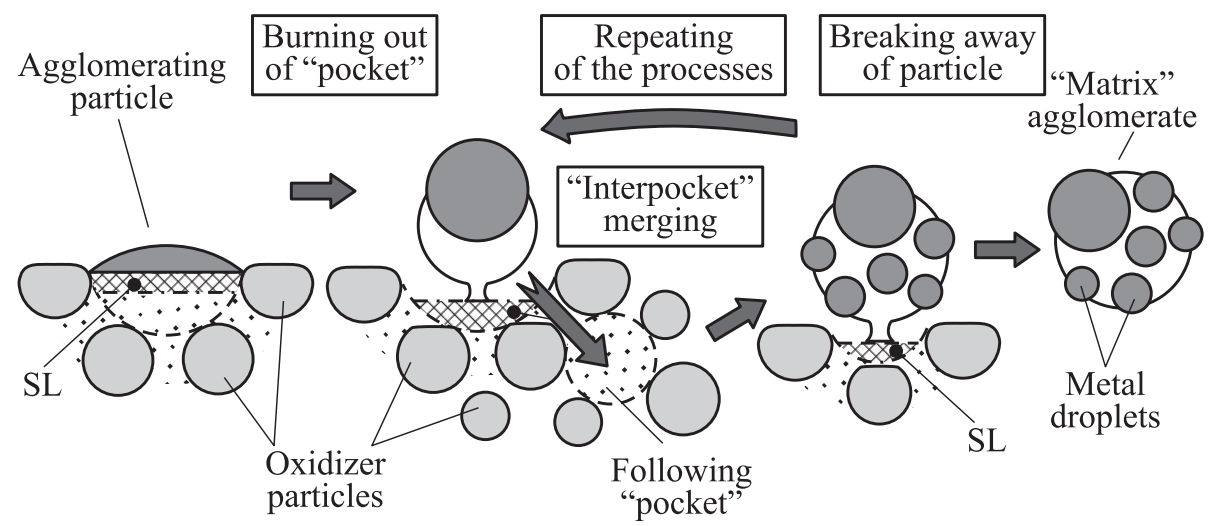

Figure 3 Scheme of the "interpocket" agglomeration mechanism

needed for burn out of the "pocket." If the degree of nonequilibrium state of the particle is more high that separation forces are capable of performing a work on its separating from SL elements, breaking away of the particle is implemented. This corresponds to the "pocket" mechanism of agglomeration.

If at burning out of "pocket" the breaking away of the particle does not occur, the particle is moving along the "interpocket bridge" into the following "pocket" and merging with its substance ("interpocket" merge). This process can occur many times, herewith size of the particle increases significantly (Fig. 3). Unlike the "pocket" agglomeration mechanism the residence time of particle staying in the II equilibrium state is comparable with the time needed for burn out of "pockets." Moreover, agglomerates forming on the "interpocket" mechanism can have "matrix" structure [34].

It was established experimentally that realization of "pocket" or "interpocket" agglomeration mechanism depends on chamber pressure. 


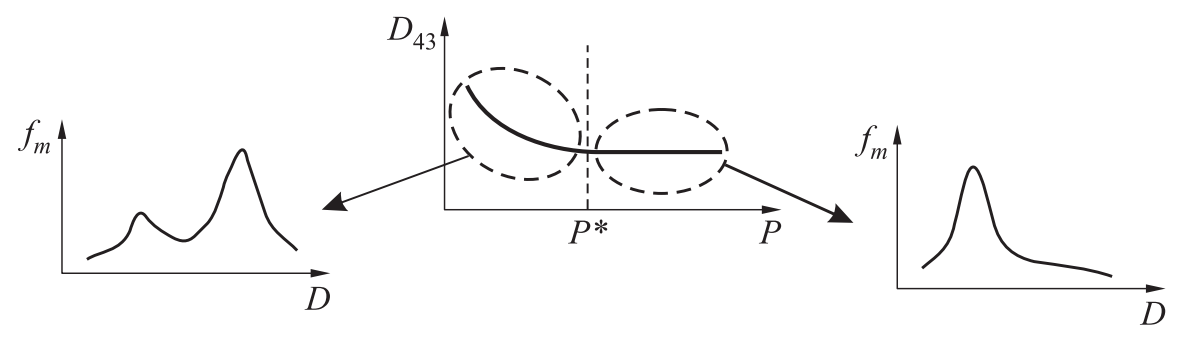

Figure 4 Dependence of the size parameters of agglomerates on the chamber pressure $P$

In the low-pressure domain, the agglomerates are forming by both the "pocket" and "interpocket" mechanisms while in the high-pressure domain agglomerates are forming mainly by the "pocket" mechanism of agglomeration [34]. It results in bimodal density distribution function of agglomerate sizes in lowpressure domain and unimodal density distribution function of agglomerate sizes in high-pressure domain (Fig. 4). Value of transition pressure $P^{*}$ is nearly 2 $4 \mathrm{MPa}$.

\subsection{Evolution of Agglomerating Particles}

It was established that agglomerating particles located on the outer SL surface in the II equilibrium state undergoing a set of physical and chemical transformations leading to chemical composition, structure, and temperature changes, i. e., particles participate in the evolution process.

Results of visualization provide obtaining of the general representations about properties of agglomerating particles on a surface of burning propellant. They consist in the following (see, for example, Fig. 5):

- agglomerating particles have higher temperature in comparison with an environment, i. e., they are burning;

- particles consist of drops of metal and its oxide. Connection of particles with a surface of burning propellant is carried out by means of oxide; and

- outflow of gaseous products from a surface of metal free of oxide and periodic change of the size of particles are possible.

The results of chemical analysis of condensed combustion products have allowed to draw a conclusion that reduction of parameter $Z_{m}^{a}$ and increasing of parameter $\eta$ are connected with combustion agglomerating metal in gas-phase mode with formation of smoke oxide particles. 


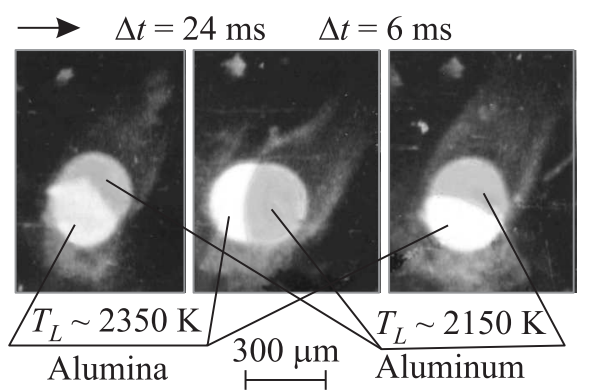

(a)

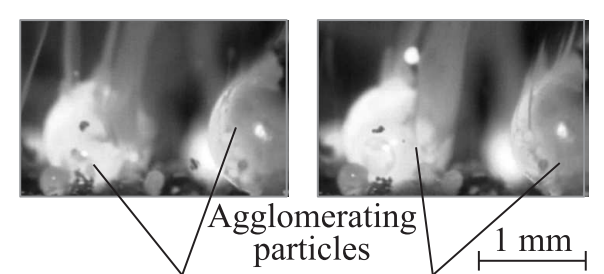

$\Delta t=1.2 \mathrm{~ms}$

Figure 5 Filming of surface layer of burning AP-based [34] (a) and AN-based [37] (b): $P=0.1 \mathrm{MPa} ; T_{L}$ - luminance temperature

The results of research of burning an acceleration field have allowed to draw a similar conclusion: agglomerating particles burn down essential in gas-phase mode. The mass of metal of agglomerates in conditions of tensile accelerations

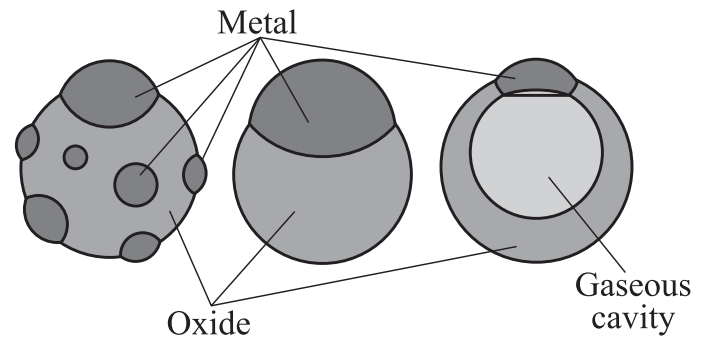

(a) (b)

(c) grows, reaching some limiting value. This is because accelerations affect on duration of evolution process [40].

As a result of the evolution on the surface of burning propellant, the agglomerates of different types are formed. The agglomerates consist of drops of metal and oxide and can have gas inclusions. The form of drops surfaces (correct, spherical) gives the basis to speak that agglomerates represent the systems, which are being in equilibri-

Figure 6 Schematic illustration of observed agglomerate types: (a) "matrix" agglomerate; $(b)$ agglomerate with "cap" oxide; and $(c)$ "hollow" agglomerate um state. By the present time, three basic types of agglomerates are established
for considered propellants: "matrix," "cap oxide," and "hollow" agglomerates $[1$, for considered propellants: "matrix," "cap oxide," and "hollow" agglomerates [1, $2,8]^{*}$. The internal structure of each type of agglomerates is schematically illustrated in Fig. 6.

As a rule, "hollow" agglomerates are fixed incidentally; however, they can become the basic type of agglomerates (AN-based propellants at use of nanoaluminum) $[1,2]$.

*The agglomerates representing a particle of metal with the oxide layer are fixed only for propellants on the base of nanoaluminum when initial particles of metal form the connected structures [37]. 


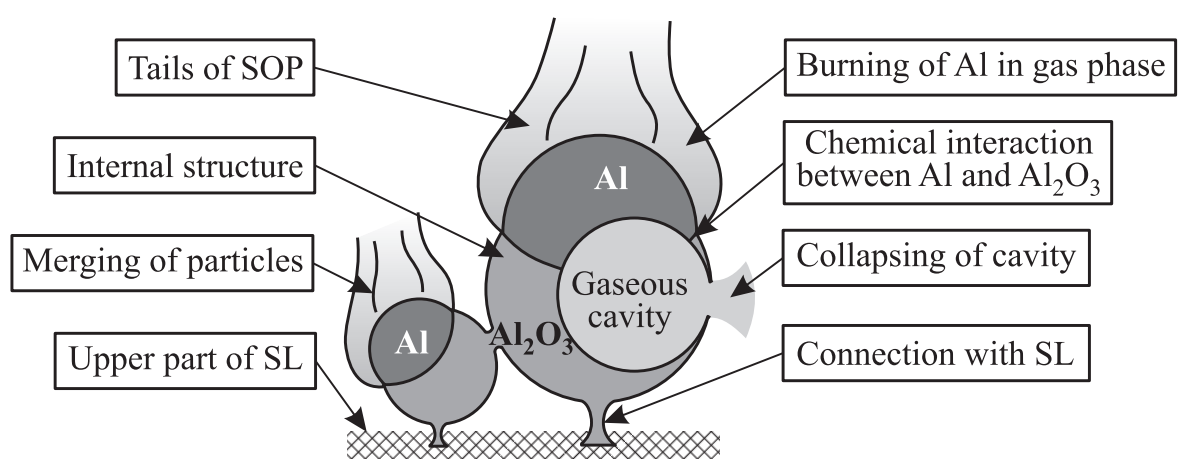

Figure 7 Scheme of evolution process of agglomerating particles on propellant surface

It is possible to offer the following set of mutually connected phenomena making evolution process (which are shown schematically in Fig. 7):

- gas-phase combustion of metal. Combustion in this mode results in formation of SOP and reduction of metal content in agglomerating particles, and later - in agglomerates (decrease of parameters $Z_{m}$ и $Z_{m}^{a}$ );

- chemical interaction condensed of $\mathrm{Al}$ and $\mathrm{Al}_{2} \mathrm{O}_{3}$. This phenomenon results in formation of gaseous products which form gas cavities inside agglomerating particles. Periodic collapses of these cavities lead to oxide removal and reduction of oxide value in agglomerates (reduction of parameter $Z_{m}^{\text {ox }}$ );

- change of particles structure. Changes in particles structure are connected with changes in fractions of metal and oxide drops, as well as gas bubble. Parameters of the structure are determined, mainly, by surface properties of particles components; and

- feeding and merging of agglomerating particles. Connection of agglomerating particle with the SL leads to enlargement of particles at the expense of taking up SL "substance" and merging of adjacent particles. Obviously, the particles enlargement depends on duration of the evolution process.

Duration of the evolution process of the agglomerating particle depends on its residence time on the SL surface in the II equilibrium state, which, in turn, depends on agglomeration mechanism. As mentioned above, for the "pocket" mechanism this time is significantly less than the time needed for burnout of the "pocket," while for the "interpocket" mechanism, it is comparable with the time needed for burnout of "pockets." 


\subsection{Breakaway of Agglomerating Particles}

Possibility of the breakaway of agglomerating particles is connected with capability of separation forces to perform a work on separating the particle from the SL elements. In other words, the breakaway of the particle is connected with its impossibility to maintain the equilibrium state in specific conditions (at action of aerodynamic and mass forces). It is considered that the following causes lead to the breakaway of agglomerating particles [24]:

- dynamism of the burning process. The breakaway takes place in the case if the speed of the moving oxidizer particle is greater in comparison with the speed of spreading of agglomerating particle substance, external forces are able to perform work on separating the particle from the SL. This situation takes place at the "pocket" mechanism implementation; and

- insufficient contact area. In the case of large particles, reduction of contact area leads to impossibility maintaining the equilibrium state of particle. This situation is typical for the "interpocket" mechanism.

\section{MODELING OF THE AGGLOMERATION PROCESS}

Creation of the mathematical description of simultaneously proceeding interconnected processes represents rather toilful problem. In this case, the approach based on decomposition of complex process of evolution on the separate simple phenomena, their modeling, and synthesis of the obtained decisions is justified. Thus, the results of the analysis of one individual model are the entrance data for another model. Such approach is more simple from the point of view of the mathematical description and favorable in practical application. Further earlier created and again developed models are considered.

\subsection{Model of Composite Solid Propellant Structure}

The model of composite solid propellant structure [41] ensures determination of the size distribution functions of "pockets" and of oxidizer particles forming each "pocket" size. Moreover, the model allows determining the fraction of the "pockets" in propellant. Thus, it is possible to determine the fraction of initial metal in propellant used to form agglomerates.

Main assumptions of the model are:

- "pocket" is formed by comparable oxidizer particles; and

- spatial distribution of oxidizer particles determined by random process. 
Input parameters of the model are:

- mass fractions of propellant components; and

- size distribution function of oxidizer particles.

The model provides determination of:

- mass fraction of "pockets" in propellant $-g_{p}$;

- size distribution function of "pockets" - $f\left(D_{p}\right)$; and

- conditional size distribution function of oxidizer particles $-f\left(D_{\mathrm{ox}} / D_{p}\right)$.

\subsection{Model of Formation of Agglomerates Size}

The model of formation of agglomerates size [24] allows to determine the size distribution function of agglomerates. The model includes modeling of particles breaking away from SL which, in turn, ensures determination of agglomeration mechanism depending on propellant composition and chamber pressure.

Input parameters are:

- size distribution function of "pockets" $-f\left(D_{p}\right)$;

- dependence of propellant burning rate on pressure; and

- environmental conditions.

Output parameters are:

- mass function of size distribution density of agglomerates $-f_{m}(D)$; and

- agglomeration mechanism — "pocket," "interpocket."

\subsection{Model of Agglomerating Particle Evolution}

The model of single agglomerating particle evolution provides predicting changes in properties of particle during its residence on the SL. This model is developed for the first time.

In turn, the evolution model is a combination of the following particular models. 


\subsubsection{Model of structure of agglomerating particles}

Generally, agglomerating particle consists of drops of metal, oxide, and gas cavities. The form and a relative positioning of these objects are determined from the following conditions:

- mechanical balance on interfaces of phases; and

- accommodations of the set quantities of substance in volumes, which are limited by spherical surfaces.

As can be concluded from analysis of equilibrium position model of the drop [24], the influence of aerodynamic and mass forces and also jointing with SL can be neglected.

The specified conditions are described by system of the transcendental algebraic equations. These equations also make the model of structure. The entrance data of the model are values of a superficial tension on interfaces of phases as functions of temperature, quantities of substances of agglomerate and its type. The superficial properties can be determined according to [5].

The section of typical agglomerate is shown in Fig. 8.

For the agglomerate presented in

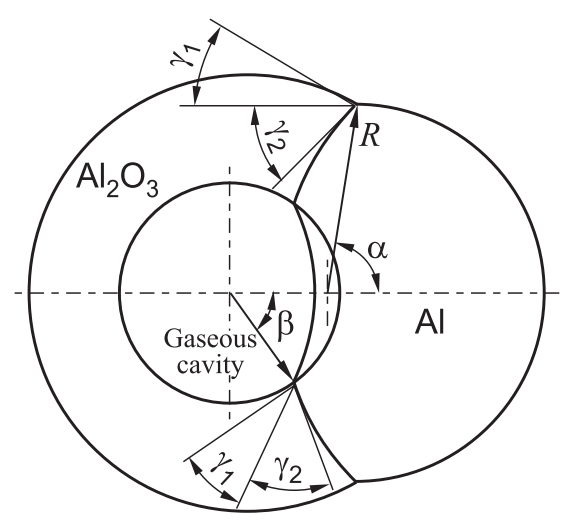

Figure 8 Section of agglomerate consisting of drops $\mathrm{Al}$ and $\mathrm{Al}_{2} \mathrm{O}_{3}$ and having a gaseous cavity; $\gamma_{1}$ and $\gamma_{2}$ - wetting angles; $R$ - radius of metal droplet surface Fig. 8, is possible to write the system of three equations:

$$
\left.\begin{array}{l}
V_{m}=f_{m}(\alpha, \beta, R) ; \\
V_{\mathrm{ox}}=f_{\mathrm{ox}}(\alpha, \beta, R) ; \\
V_{g}=f_{g}(\beta, R) .
\end{array}\right\}
$$

Here, $V_{m}, V_{\text {ox }}$ and $V_{g}$ are the volumes $\left(\mathrm{m}^{3}\right)$ of metal, oxide droplets, and gaseous cavity, respectively; $f_{m}, f_{\text {ox }}$, and $f_{g}$ are the functions of geometric parameters; $\alpha$ and $\beta$ are the angles; and $R$ is the radius of metal droplet surface $(\mathrm{m})$.

Equations (1) can be extended to any number of droplets and cavities. Generally, the developed model allows to describe the structure of all kinds of agglomerates known to the present time. 


\subsubsection{Model of gas-phase combustion of metal}

The model is based on ideology of work [5]. The main assumptions of the model are:

- approximation of a "reduced film" is used;

- all reactions take place in equilibrium mode;

- burning process is controlled by diffusion;

- burning process is quasi-stationary, isobaric, and axisymmetric; and

- oxidizing components from the environment are $\mathrm{H}_{2} \mathrm{O}, \mathrm{O}_{2}$, and $\mathrm{CO}_{2}$, and its relative content is determined by the model of environmental conditions.

The model is the system of the equations describing transfer of substance and energy within the limits of burning area. The equation of mass transfer for the $i$ th component has the following form:

$$
J_{i}=-\Omega\left(\frac{\lambda_{T}}{C_{p}}\right)\left(1-\bar{P}_{i} \frac{\sum_{j=1}^{N} J_{j}}{J_{i}}\right)^{-1} \frac{d \bar{P}_{i}}{d r}
$$

Here, $J_{i}$ is the molar flux (mol/s) of the $i$ th component through area $\Omega\left(\mathrm{m}^{2}\right) ; \lambda_{T}$ is the thermal conductivity $(\mathrm{W} /(\mathrm{m} \cdot \mathrm{K})) ; C_{p}$ is the molar isobaric heat capacity $(\mathrm{J} /(\mathrm{mol} \cdot \mathrm{K})) ; \bar{P}_{i}=P_{i} / P$ where $P_{i}$ is the partial pressure of the $i$ th component $(\mathrm{Pa})$ and $P$ is the chamber pressure $(\mathrm{Pa}) ; N$ is the number of components transported through area $\Omega$ including the $i$ th component; and $r$ is the axial coordinate. Integration of Eq. (2) gives 6 equations describing diffusion of oxidizer, metal vapor, and dissociated oxide.

Energy transport is described by equations of energy balance having the form:

$$
\sum_{i=1}^{n} J_{i}^{+} H_{i}-\sum_{j=1}^{n} J_{j}^{-} H_{j}+Q_{s}+Q_{\text {cond }}^{+}-Q_{\text {cond }}^{-}+Q_{\text {rad }}^{+}-Q_{\text {rad }}^{-}=0 .
$$

Here, index "+" means "inflow," index "-" means "outflow;" $H_{i}$ is the molar enthalpy of the $i$ th component $(\mathrm{J} / \mathrm{mol}) ; Q_{s}$ is the heat flux $(\mathrm{W})$ consumed by interaction between $\mathrm{Al}$ and $\mathrm{Al}_{2} \mathrm{O}_{3} ; Q_{\text {cond }}$ is the conductive heat flux (W); and $Q_{\text {rad }}$ is the radiant heat flux (W). Integration of Eq. (3) gives 4 equations of energy balance within burning area.

Solving the system of 6 equations of mass transfer and 4 equations of heat transfer provides determination of metal burning rate $J_{m}(\mathrm{~mol} / \mathrm{s})$ and temperature of metal particle $T_{A}(\mathrm{~K})$. 
The entrance data of model are the size of metal particle, a fraction of its surface free from oxide (these parameters are provided by the model of structure), and parameters of a stream flowing around a particle: oxidizing potential, temperature, and mass speed which are provided by the model of environmental conditions.

\subsubsection{Model of chemical interaction of condensed $\mathrm{Al}$ and $\mathrm{Al}_{2} \mathrm{O}_{3}$}

It is shown in [5] that in the certain conditions, the interaction of condensed $\mathrm{Al}$ and $\mathrm{Al}_{2} \mathrm{O}_{3}$ with formation of gaseous products (basically, $\mathrm{Al}$ and $\mathrm{Al}_{2} \mathrm{O}$ ) is possible:

$$
\mathrm{Al}_{2} \mathrm{O}_{3}^{c}+x_{1} \mathrm{Al}^{c} \leftrightarrow 3 \mathrm{Al}_{2} \mathrm{O}^{g}+x_{2} \mathrm{Al}^{g} .
$$

Coefficients $x_{1}$ and $x_{2}$ depend on chamber pressure $P$. Superscripts $c$ means "condensed" and $g$ — "gaseous."

It is remarkable that the equilibrium temperature of reaction is much lower than the temperature of boiling metal and oxide. The consequences of realization of the specified process depend on a competition of speeds of formation of gaseous products and their condensation. It is assumed that reaction takes place at equilibrium mode.

Evaporation rate of condensed $\mathrm{Al}_{2} \mathrm{O}_{3} \omega_{1}\left(\mathrm{~mol} /\left(\mathrm{s} \cdot \mathrm{m}^{2}\right)\right)$ is described by the following expression:

$$
\omega_{1}=A_{1} \exp \left(-\frac{E_{a}}{R_{0} T_{A}}\right) \frac{1}{\sqrt{T_{A}}}\left(P_{s}-P\right) .
$$

Here, $A_{1}$ is the coefficient depending on mixture properties; $E_{a}$ is the activation energy; $R_{0}=8.314 \mathrm{~J} /(\mathrm{mol} \cdot \mathrm{K})$ is the universal gas constant; and $P_{s}$ is the pressure of saturated vapor of the reaction products $(\mathrm{Pa})$. Pressure $P_{s}$ is determined by Clausius-Clapeyron relation:

$$
P_{s}\left(T_{A}\right)=P^{*} \exp \left[\frac{L_{s}}{R_{0}}\left(\frac{1}{T_{s}^{*}}-\frac{1}{T_{A}}\right)\right] .
$$

Here, $P^{*}=100 \mathrm{kPa}$ is the standard pressure; $L_{s}$ is the evaporation heat of 1 mole of mixture $(\mathrm{J} / \mathrm{mole})$; and $T_{s}^{*}$ is the evaporation temperature $(\mathrm{K})$ of mixture at standard conditions.

The rate of formation of condensed $\mathrm{Al}_{2} \mathrm{O}_{3} \omega_{2}\left(\mathrm{~mol} /\left(\mathrm{s} \cdot \mathrm{m}^{2}\right)\right)$ is described by the expression:

$$
\omega_{2}=A_{1} \exp \left(-\frac{E_{a}}{R_{0} T_{A}}\right) \frac{1}{\sqrt{T_{A}}}(P-0) .
$$


The molar fluxes of reacting oxide $J_{\mathrm{ox}}$, metal $J_{m}^{s}$, and the rate of gaseous bubble growth $J_{g}(\mathrm{~mol} / \mathrm{s})$ is described by the following expressions:

$$
\begin{aligned}
J_{\mathrm{ox}} & =\Omega_{1} \omega_{1}-\Omega_{2} \omega_{2} ; \\
J_{m}^{s} & =x_{1} J_{\mathrm{ox}} \\
J_{g} & =\left(3+x_{2}\right) J_{\mathrm{ox}} .
\end{aligned}
$$

Evaporation takes place at area $\Omega_{1}$ located on interphase boundary of the condensed $\mathrm{Al}_{2} \mathrm{O}_{3}$ and $\mathrm{Al}$. Condensation of gaseous products takes place at area $\Omega_{2}$ on a part of surface of the bubble.

From the model of structure of agglomerating particle, it can be concluded that the bubble is located mostly inside the oxide drop. If the bubble exceeds the limits of oxide drop, it is collapsing and gaseous products are removed outside. The results of paper [5] have been used for definition of parameters $A_{1}$ and $E_{a}$ which are necessary for the numerical analysis of model.

The model allows to determine the resulting speed of gas formation and the rate of removal of oxide and metal. The entrance data of model are the data of internal structure and temperature of agglomerating particle.

\subsubsection{Model of particles feeding and merging}

It is considered that the process of particles merge is a "fast" process. The merge is carried out within the limits of the "pocket" at realization of the "pocket" mechanism. "Interpocket" merge takes place at realization of other mechanism — "interpocket."

Agglomerating particles staying in the II equilibrium state at the surface of burning propellant are fed by liquid metal and oxide in composition of the SL. Its rate depends mainly on decomposition rate of the "pocket" substance.

Both processes of merging and feeding can be described by the following scheme. It is considered that liquid aluminum formed in unit of time during decomposition of "pocket" became a part of the SL. Some part of metal burns in heterogeneous mode inside the SL and forms liquid oxide. As soon as decomposition of the upper part of SL occurs, the condensed substances $\mathrm{Al}$ and $\mathrm{Al}_{2} \mathrm{O}_{3}$ feed the agglomerating particle. Herewith, it is assumed that the rate of decomposition of "pocket" substance and the rate of decomposition of the upper part of the SL have the same value. So, the ratio between condensed $\mathrm{Al}$ and $\mathrm{Al}_{2} \mathrm{O}_{3} \eta_{\mathrm{SL}}$ in the SL is constant in time. The mass flux of substance feeding the agglomerating particle $j_{s}(\mathrm{~kg} / \mathrm{s})$ is described by the expression:

$$
j_{s}=u_{\mathrm{dec}} \Omega_{\mathrm{p}-\mathrm{SL}} \frac{k_{m}}{k_{m} / \rho_{m}+k_{\mathrm{bin}} / \rho_{\mathrm{bin}}}\left(1+\frac{3 \mu_{\mathrm{O}} \eta_{\mathrm{SL}}}{2 \mu_{\mathrm{Al}}+3 \mu_{\mathrm{O}}\left(1-\eta_{\mathrm{SL}}\right)}\right) .
$$


Here, $u_{\mathrm{dec}}$ is the rate of "pocket" decomposition $(\mathrm{m} / \mathrm{s}) ; \Omega_{\mathrm{p}-\mathrm{SL}}$ is the area of contact of a particle in the II equilibrium state with $\mathrm{SL}\left(\mathrm{m}^{2}\right) ; k_{m}$ and $k_{\text {bin }}$ are the mass fractions of metal and binder in propellant composition, respectively; $\rho_{m}=2700 \mathrm{~kg} / \mathrm{m}^{3}$ is the density of aluminum; $\rho_{\text {bin }}$ is the density of binder $\left(\mathrm{kg} / \mathrm{m}^{3}\right) ; \mu_{\mathrm{Al}}=0.027 \mathrm{~kg} / \mathrm{mol}$ is the molar mass of $\mathrm{Al} ;$ and $\mu_{\mathrm{O}}=0.016 \mathrm{~kg} / \mathrm{mol}$ is the molar mass of atomic oxygen.

Ratio between metal and oxide in the SL $\eta_{\mathrm{SL}}$ is determined using the experimental data for combustion of various propellants in acceleration field. If tensile accelerations exceed some limiting value, then duration of evolution is negligible, and chemical composition of agglomerates corresponds to the ratio between metal and oxide in the SL. It is shown that in the absence of accelerations, up to $25 \%$ (mass) of metal in agglomerating particles burn in gas-phase mode at their evolution [40]. Thus, it was assumed that at zero accelerations, evolution duration at realization of the "pocket" mechanism is determined by the time needed for burnout of $25 \%$ (mass) of metal.

\subsubsection{Model of smoke oxide particles deposition}

Presence of SOPs in the gaseous flow leads to theoretical capability of their deposition on the surface of agglomerating particle. This phenomenon could result in increasing of oxide mass in the particle.

Based on mathematical description in [5], the model of SOPs deposition was implemented. The analysis of the model allowed to make conclusion that minor part of the SOPs in the flow is depositing on the surface of agglomerating particle. Increasing of oxide fraction in the agglomerating particle is insignificant. The analysis was made in wide range of agglomerating particle and SOPs sizes. Thus, the phenomenon of SOPs deposition can be neglected at the evolution modeling.

Combination of listed above models except the SOPs deposition model represents the model of agglomerating particle evolution on the surface of burning propellant. Interdependence between particular models is shown in Fig. 9.

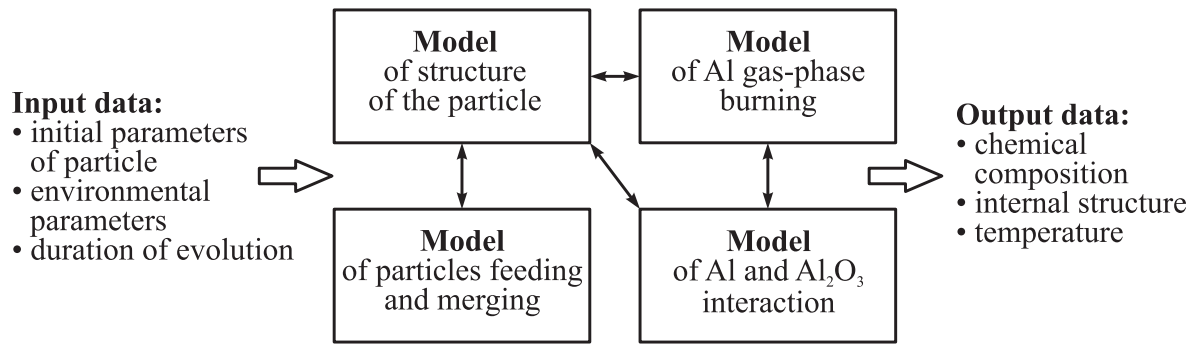

Figure 9 Block scheme of the evolution model: interdependence between particular models 
The model of the evolution provides determination of chemical composition, internal structure, and temperature of agglomerating particle. Input parameters are: initial parameters of agglomerating particle, environmental parameters, and duration of evolution. Initial size of the particle is provided by the model of formation of agglomerates size. Initial chemical composition corresponds to composition of SL. Evolution time duration is determined by the residence time of agglomerating particle staying in the II equilibrium state.

\subsection{Model of Environmental Conditions}

Environmental conditions affect on the processes taking place at evolution of agglomerating particle. Gas-phase burning rate of metal depends significantly on properties of gaseous flow around the particle. Most important of them are oxidizing potential, temperature, and mass velocity.

Spatial area situated close to burning propellant surface is characterized by high level of nonequilibrium processes. Schematization and simplification are needed. The conditions around the agglomerating particle differ significantly at "pocket" and "interpocket" mechanisms of agglomeration.

"Pocket" mechanism. At burnout of the "pocket," new oxidizer particles appear which are produced of oxidizing components. Thus, the agglomerating particle is staying mainly in the flow of oxidizer decomposition products.

"Interpocket" mechanism. The agglomerating particle is staying in the II equilibrium state during the time of "pocket" burning. Herewith, it is situated mainly in the flow of binder decomposition products.

Oxidizer and binder decomposition products interact and form the diffusive flame at some distance from the surface of burning propellant. Local composition and properties of interaction products are determined by composition and structure of considered local propellant area - of the "pocket" and surrounding "interpocket bridges" and oxidizer particles. For example, formation of local combustion products using the AP oxidizer can be written by the following reaction:

$$
\begin{aligned}
& A \mathrm{C}_{w} \mathrm{H}_{x} \mathrm{O}_{y} \mathrm{~N}_{z}^{c}+\mathrm{NH}_{4} \mathrm{ClO}_{4}^{c}+B \mathrm{Al}^{c} \rightarrow \\
& \quad \rightarrow \mathrm{Al}_{2} \mathrm{O}_{3}^{c}+\mathrm{Al}^{c}+\mathrm{O}_{2}+\mathrm{H}_{2}+\mathrm{OH}+\mathrm{H}_{2} \mathrm{O}+\mathrm{HCl}+\mathrm{N}_{2}+\mathrm{NO}+\mathrm{CO}+\cdots .
\end{aligned}
$$

Indexes $w, x, y$, and $z$ depend on binder type. Coefficients $A$ and $B$ depend on composition of the considered local propellant area. Superscript $c$ means "condensed." Partial pressures (or molar composition) of reaction products and their temperature are determined by thermodynamic evaluations. The oxidizing potential is formed by partial pressures of oxidizers $\mathrm{O}_{2}, \mathrm{H}_{2} \mathrm{O}$, and $\mathrm{CO}_{2}$. Parameters of heat and mass transfer such as diffusivity $D$, thermal conductivity $\lambda_{T}$, and dynamic viscosity $\mu$ are determined by the methods described in [42]. 
It is assumed the following: the parameters depend on distance from the surface of burning propellant and vary linearly. The height of formation of the local equilibrium combustion products is determined by solving the BurkeSchumann's problem [43]. (The conditions of gas-phase metal burning can be estimated for known size particle.)

It is assumed that mass velocity of gaseous flow does not depend on the distance from the surface of the burning propellant. Its value is connected with propellant burning rate by the continuity equation.

According to the statements described above, the model of environmental conditions is developed. Input data of the model are the composition and structure of composite solid propellant, its burning rate, and chamber pressure. The model allows to determine composition, temperature, mass, and heat transfer parameters of gaseous flow around the agglomerating particle.

\subsection{The Synthesis of Individual Models}

According to accepted methods, the model of the agglomeration process is the result of synthesis of the models presented above.

The model of agglomerates formation is described below:

1. Based on the data of propellant composition, modeling of solid propellant structure is performed, and the size distribution function of "pockets" is found.

2. Based on the data of propellant burning rate and external conditions, modeling size distribution function of agglomerates is performed. Moreover, collecting the data of quantity and sizes of "pockets" participating in each agglomerate formation is carried out.

3. For the agglomerate having any size $D_{i}$, size distribution function of "pockets" participating in its formation is found based on the collected data of the quantity and sizes of "pockets" participating in each particle formation (see above). This function contains the conditions of breakaway of considered particle in implicit form.

4. Based on previously obtained data, modeling of environmental conditions and agglomerating particle evolution is carried out. Herewith, the size of "pocket" is selected randomly based on the size distribution function obtained on step 3. If agglomerate is formed only from one "pocket," the "pocket" mechanism of agglomeration is implemented and evolution duration is determined on the base of experimental data [40]. If agglomerate is formed from few "pockets," the "interpocket" mechanism is implemented and the evolution modeling is performed until the agglomerating particle reaches $D_{i}$ in size. 
5. Varying $D_{i}$ and repeating the modeling on steps 3 and 4, this scheme can be expanded to the full range of agglomerate sizes.

The algorithm described above is presented in Fig. 10.

Thus, the developed model provides definition of the following characteristics of agglomerates:

- the function of size distribution of agglomerates (it is supposed that evolution process does not essentially influence the size of agglomerates);

- the fraction of initial metal in the propellant used to form agglomerates (parameter $Z_{m}^{a}$ ). The fraction of "pockets" in propellant is determined primarily. This value allows to find quantity of the metal fuel participating in agglomeration. Then the fraction of this metal, which is burning down in gas-phase mode, is considered; and

- the parameters of chemical composition and structure of agglomerates of the various sizes.

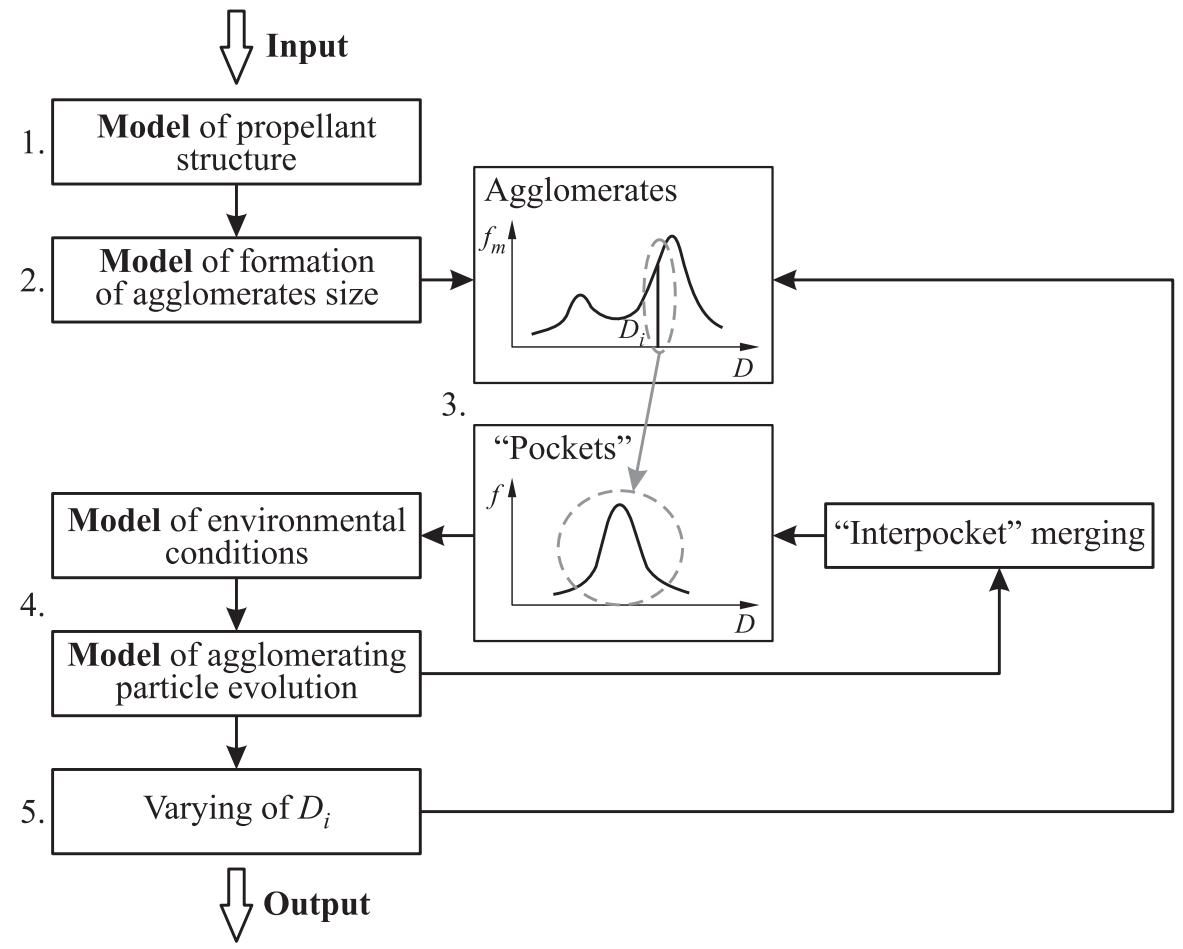

Figure 10 Block scheme of the synthesis of different models 


\section{RESULTS OF THE MODEL ANALYSIS}

The results of the numerical analysis of formation of size agglomerates model correspond to the experimentally fixed features: agglomerates are formed mainly by the "pocket" mechanism in the high-pressure domain and by both the "pocket" and "interpocket" mechanisms in the low-pressure domain. As a consequence, there is the unimodal character of the density distribution function of agglomerate sizes in the high-pressure domain (Fig. 11a) and the bimodal character of this function in the low-pressure domain (Fig. 11b)*.

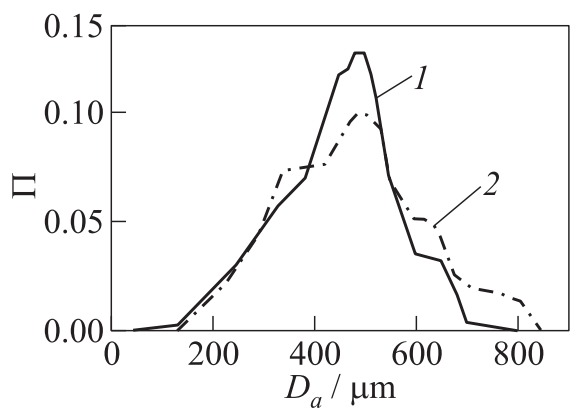

(a)

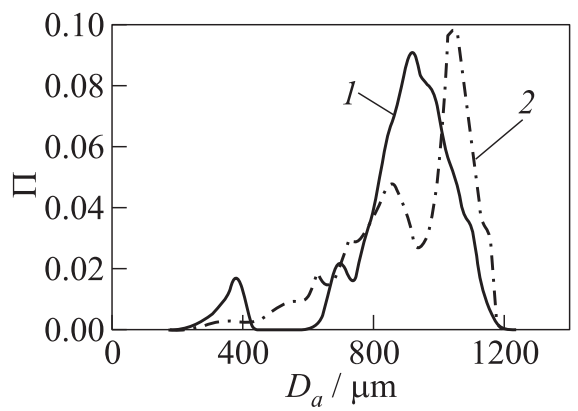

(b)

Figure 11 Smoothed density distribution functions of agglomerate sizes at pressures $P=6.2(a)$ and $0.4 \mathrm{MPa}(b)[24]: 1$ - calculation and 2 - experiment

Determination of the agglomerates chemical composition (Fig. 12a) results in the following:

- the discrepancy between the calculated and experimentally obtained data can be explained by the following: at low pressures, the changes in the propellant burning rate lead to changes in the SL structure (size and amount of pores), which, in turn, influence the parameters of the heterogeneous mode of metal burning. Thus, for adequate modeling of agglomerates chemical composition in low-pressure domain, it is necessary to develop the mathematical model of the heterogeneous combustion of metal inside the SL; and

- in the high-pressure domain, the agglomerates chemical composition almost does not depend on the chamber pressure. It is connected with constancy of the SL chemical composition and significantly shorter duration of the evolution process at the "pocket" mechanism domination. Composition of SL also does not depend on pressure.

*The model propellant is considered. Bimodal oxidizer: $70 \%$ coarse $(500-700 \mu \mathrm{m})+30 \%$ fine $(16-315 \mu \mathrm{m})$. 


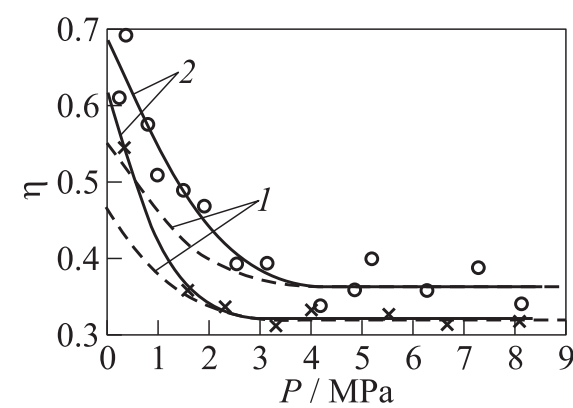

(a)

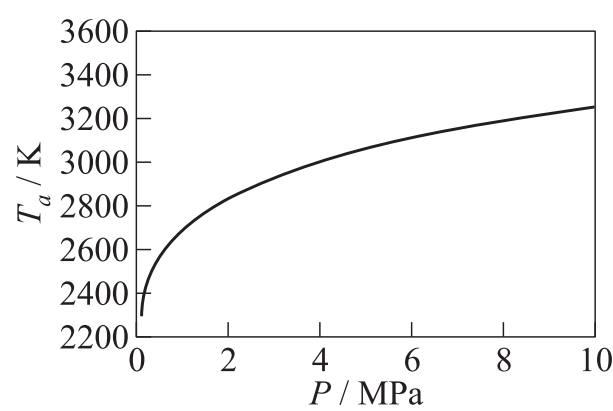

(b)

Figure 12 Dependencies of the mass fraction of oxide in agglomerates $\eta(a)$ and agglomerate temperature $T_{a}(b)$ on chamber pressure $P$ for two model propellants that differ in size of oxidizer particles: 1 - calculation and 2 - experiment

Modeling of the evolution process allowed to draw the following:

- it is shown that formation of "hollow" agglomerate is the consequence of change of structure agglomerating particle at the last stages of this process;

- it is shown that periodic change of the size agglomerating particles is the consequence of realization of the mechanism of carrying out of gaseous products at interaction of condensed $\mathrm{Al}$ and $\mathrm{Al}_{2} \mathrm{O}_{3}$; and

- it is shown that combustion of metal in gas-phase mode is the factor, which influences essentially the properties of condensed combustion products at the surface of the burning propellant.

The model of the evolution of agglomerating particles allows also determining the temperature of particles (Fig. 12b). According to this model, the particles temperature corresponds to the temperature of equilibrium evaporation of metal at gas-phase burning.

The results of the modeling of the agglomerates structure and their comparison with experimental data allowed to make the conclusion of adequacy of the mathematical model (Fig. 13). The model allows to describe the structure of all the agglomerates types including "matrix" and "hollow" agglomerates.

\section{CONCLUDING REMARKS}

The basic results of the present work can be defined as follows:

- the physical picture of the agglomerating process is developed for propellants based on AP, ADN, HMX, inactive binder, and nanoaluminum; 

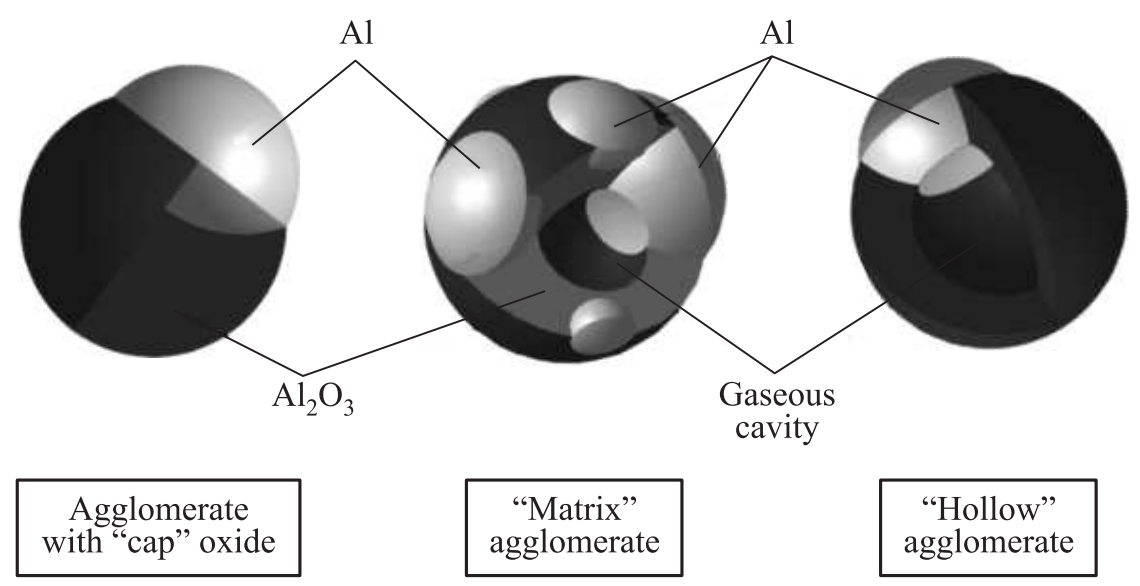

(a)

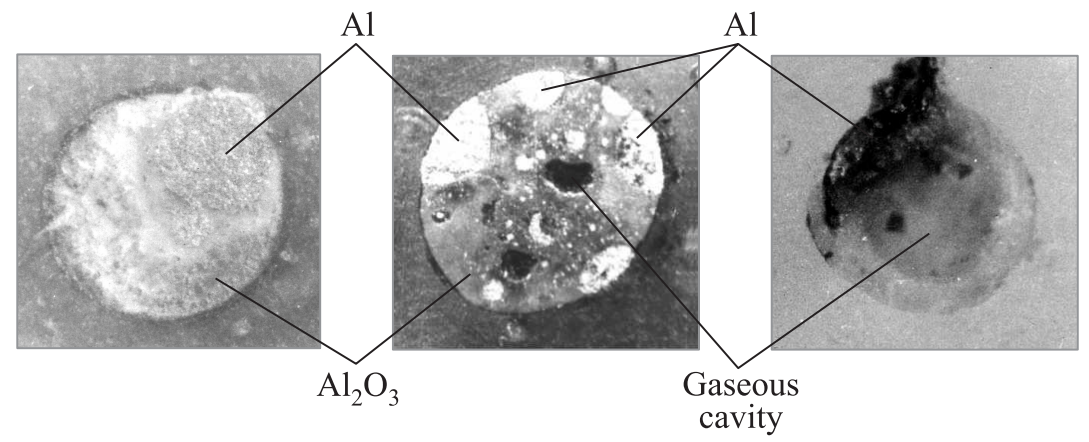

(b)

Figure 13 Results of modeling of agglomerate structure $(a)$ and comparison with experimental data $(b)$

- the model of evolution of agglomerating particles on surface of burning propellant is developed;

- the analysis and synthesis of mathematical models is carried out that provides prediction of the agglomeration process parameters;

- it is shown that presence of the evolution process of agglomerating particles influence essentially the properties of the condensed combustion products; and

- the conclusion on the need for the model of metal combustion in heterogeneous mode inside SL is made. 
The obtained results indicate the rightfulness of the accepted physical picture and it is shown that solution of the agglomeration modeling problem is fundamentally possible for other types of metalized solid propellants.

Further work will be directed on development of the mathematical model of metal combustion in heterogeneous mode inside SL. It is assumed that such model will allow predicting the chemical composition of agglomerate with higher accuracy as well as describe the effect of the agglomeration process on the propellant burning law.

\section{REFERENCES}

1. Babuk, V.A., V.A. Vasilyev, A.A. Glebov, I. N. Dolotkazin, M. Galeotta, and L. T. DeLuca. 2004. Combustion mechanisms of AN-based aluminized solid rocket propellants. In: Novel energetic materials and applications. Eds. L. T. DeLuca, L. Galfetti, and R. A. Pesce-Rodriguez. Bergamo, Italy: Grafiche GSS. Paper 44.

2. Babuk, V., I. Dolotkazin, A. Gamsov, A. Glebov, L. T. DeLuca, and L. Galfetti. 2009. Nanoaluminum as a solid propellant fuel. J. Propul. Power 25(2):482-89.

3. Dupays, J., Y. Fabignon, P. Villedieu, G. Lavergne, and J. L. Estivalezes. 2000. Some aspects of two-phase flows in solid-propellant rocket motors. In: Solid propellant chemistry, combustion, and motor interior ballistics. Eds. V. Yang, T. B. Brill, and W.Z. Ren. Progress in astronautics and aeronautics ser. Reston, VA: AIAA. 185(Ch. 2.21):859-83.

4. Shishkov, A. A., S. D. Panin, and B. V. Rumyantsev. 1988. Working processes in solid rocket motors. Handbook. M.: Mashinostroenie. 207-11. [In Russian.]

5. Babuk, V. A., and V.A. Vasilyev. 2002. Model of aluminum agglomerate evolution in combustion products of solid rocket propellant. J. Propul. Power 18(4):814-24.

6. Beckstead, M. W. 2010. An overview of aluminum agglomeration modeling. 50th Annual Israeli Aerospace Sciences Conference Proceedings. Tel Aviv. 1-28.

7. Bandera, A. 2009. Combustion of metallized solid rocket propellants and motor performance. Ph.D. Thesis. SPLab, Politecnico di Milano. 1-48.

8. Maggi, F., L.T. De Luca, and T.L. Jackson. 2009. Using statistics for agglomerate prediction in aluminized rocket propellants. 3rd European Conference for Aerospace Sciences Proceedings. Versailles, France. Paper 308.

9. Crump, J.E. 1966. Aluminum combustion in composite propellants. 2nd ICRPG Combustion Conference, CPIA. Publ. 105. I:321-29.

10. Beckstead, M. W. 1977. A model for solid propellant combustion. 14th JANNAF Combustion Meeting, CPIA. 1(292):281-306.

11. Cohen, N.S. 1981. A pocket model for aluminum agglomeration in composite propellants. AIAA Paper No. 1585.

12. Grigor'ev, V.G., K.P. Kutsenogii, and V.E. Zarko. 1981. Model of aluminum agglomeration during the combustion of a composite propellant. Combust. Explosion Shock Waves 17(4):356-62. 
13. Gallier, S. 2009. A stochastic pocket model for aluminum agglomeration in solid propellants. Propellants Explosives Pyrotechnics 34(2):97-105.

14. Jackson, T. L., F. Najjar, and J. Buckmaster. 2005. New aluminum agglomeration models and their use in solid-propellant-rocket simulations. J. Propul. Power 21(5):925-36.

15. Gladun, V.D., Yu. V. Frolov, L. Ya. Kashporov, and G. A. Ostretsov. 1976. A model for detachment of a condensed particle from a combustion surface. Combust. Explosion Shock Waves 12(2):191-97.

16. Gladun, V. D., Yu. V. Frolov, and L. Ya. Kashporov. 1977. Coalescence of powdered aluminum particles on combustion surface of metallized compositions. Combust. Explosion Shock Waves 13(5):705-10.

17. Gany, A., L. H. Caveny, and M. Summerfield. 1978. Aluminized solid propellants burning in a rocket motor. AIAA J. 16(7):736-39.

18. Zyryanov, V. Ya. 1986. Model for predicting agglomeration in combustion of metallized systems. VIII All-Union Symposium on Combustion and Explosion Proceedings. Chernogolovka. 59-62. [In Russian.]

19. Kovalev, O. B., A. P. Petrov, and A.V. Fol'ts. 1987. Simulating aluminum powder aggregation in mixed condensed system combustion. Combust. Explosion Shock Waves 23(2):133-36.

20. Kovalev, O.B. 1989. Model of the agglomeration of aluminum in the combustion of mixed condensed systems. Combust. Explosion Shock Waves 25(1):39-48.

21. Kovalev, O. B., A. P. Petrov, and V. M. Fomin. 1993. Combustion wave structure in heterogeneous solid propellants. Combust. Explosion Shock Waves 29(3):8-16.

22. Rashkovsky, S.A. 1998. Monte Carlo simulation of aluminum agglomeration in composite solid propellants combustion. 24th Pyrotechnics Seminar (International) Proceedings. Monterey, California. 833-46.

23. Rashkovsky, S. A. 2002. Aluminum agglomeration in composite solid propellants combustion. J. Phys. IV France 12(7):453-58.

24. Babuk, V.A., I. N. Dolotkazin, and V. V. Sviridov. 2003. Simulation of agglomerate dispersion in combustion of aluminized solid propellants. Combust. Explosion Shock Waves 39(2):195-203.

25. Rashkovsky, S. A. 2004. Stochastic modeling of burning processes of the heterogeneous condensed systems. Dissertation. Moscow. 428 p. [In Russian.]

26. Tanner, M. W., M. W. Beckstead, I. L. Davis, and M. Webb. 2006. Numerical modeling of aluminum agglomeration in solid propellants using a three-dimensional particle packing model. 41st JANNAF Combustion Meeting Proceedings.

27. Srinivas, V., and S. R. Chakravarthy. 2007. Computer model of aluminum agglomeration on burning surface of composite solid propellant. J. Propul. Power 23(4):72836 .

28. Tanner, M. W., and M.W. Beckstead. 2009. Diffusion flame considerations in the prediction of aluminum agglomerate diameters. 43rd JANNAF Combustion Meeting Proceedings.

29. Sankaralingam, K., and S.R. Chakravarthy. 2000. Computer model of flamelet distribution on the burning surface of a composite solid propellant. Combust. Sci. Technol. 161(7):49-68. 
30. Massa, L., T. L. Jackson, J. Buckmaster, and M. Campbell. 2002. Proc. Combust. Inst. 29(2):2975-83.

31. Maggi, F., A. Baietta, and L. T. De Luca. 2011. Solid propellant microstructure representation: A packing code. 4th European Conference for Aerospace Sciences Proceedings. St. Petersburg, Russia.

32. Babuk, V. A., V. P. Belov, V. V. Khodosov, and G. G. Shelukhin. 1988. Study of the structure of agglomerates with combustion of aluminized mixed condensed systems. Combust. Explosion Shock Waves 24(5):52-57.

33. Babuk, V.A., V.A. Vasilyev, and M. S. Malakhov. 1999. Condensed combustion products at the burning surface of aluminized solid propellant. J. Propulsion Power 15(6):783-94.

34. Babuk, V.A., V. A. Vasilyev, and V. V. Sviridov. 2000. Formation of condensed combustion products at the burning surface of solid rocket propellant. In: Solid propellant chemistry, combustion, and motor interior ballistics. Eds. V. Yang, T. B. Brill, and W.Z. Ren. Progress in astronautics and aeronautics ser. Reston, VA: AIAA. 185:749-76.

35. Babuk, V. A., V. A. Vasilyev, and V. V. Sviridov. 2001. Propellant formulation factors and metal agglomeration in combustion of aluminized solid rocket propellant. Combust. Sci. Technol. 163:261-89.

36. Babuk, V. A., A. A. Glebov, and I. N. Dolotkazin. 2005. Burning mechanism of aluminized solid rocket propellants based on energetic binders. Propellants Explosives Pyrotechnics 30(4):281-90.

37. Babuk, V., A. Glebov, I. Dolotkazin, A. Conti, L. Galfetti, L. DeLuca, and A. Vorozhtsov. 2009. Condensed combustion products from burning of nanoaluminum-based propellants: Properties and formation mechanism. In: Progress in propulsion physics. Eds. L. DeLuca, C. Bonnal, O. Haidn, and S. Frolov. EUCASS advances in aerospace sciences book ser. 1:3-16.

38. Babuk, V.A. 2009. Properties of the surface layer and combustion behavior of metalized solid propellants. Combust. Explosion Shock Waves 45(4):486-94.

39. Babuk, V.A. 2007. Problems in studying formation of smoke oxide particles in combustion of aluminized solid propellants. Combust. Explosion Shock Waves 43(1):3845.

40. Babuk, V. A., V.A. Vasil'ev, and A. N. Potekhin. 2009. Experimental investigation of agglomeration during combustion of aluminized solid propellants in an acceleration field. Combust. Explosion Shock Waves 45(1):32-39.

41. Babuk, V. A., V. A. Vasil'ev, and V.V. Sviridov. 1999. Modeling the structure of composite solid rocket fuel. Combust. Explosion Shock Waves 35(2):144-48.

42. Glushko, V.P. 1971. Thermodynamic and thermophysical properties of combustion products. Moscow: VINITI. [In Russian.]

43. Zel'dovich, Y.B., G. I. Barenblatt, V. B. Librovich, and G. M. Makhviladze. 1980. Mathematical theory of combustion and explosion. Moscow: Nauka. 450-60. [In Russian.] 